\title{
High Resolution Observations of Sub-Kiloparsec Circumnuclear Gas in AGN
}

\author{
J. E. Conway \\ Onsala Space Observatory, Onsala, Sweden
}

\begin{abstract}
.
In recent years it has become possible to use high resolution radio interferometry and other techniques to study the sub-kiloparsec scale circumnuclear gas in the centres of Active Galactic Nuclei. This gas presumably provides the fuel source for the AGN activity and also plays a role in obscuring the direct view of the central engine from certain directions. The status of high resolution observations of the ionised, atomic and molecular phases of this circumnuclear gas are briefly reviewed.
\end{abstract}

\section{Introduction}

In the last five years there has been substantial progress in using interferometric observations to study circumnuclear gas in the inner kiloparsec of AGN. Here we review these results, concentrating on the highest resolution images from radio interferometry but considering also complementary millimetre and other data. Historically radio observations have concentrated on imaging synchrotron emission from relativistic plasmas emitted in the forms of jets. However, with increased sensitivity and spectral capabilities it has become possible to instead probe the properties of the non-relativistic gas in the centres of AGN hosts. This can be done via observations of atomic and molecular spectral absorption, maser emission, thermal continuum emission, free-free absorption, depolarisation and rotation measure mapping. In this review I discuss the current state of the observations, first in low luminosity Seyfert galaxies (in Section 2) and then in powerful radio-loud AGN (in Section 3). Finally in Section 4, the properties of the two classes are briefly compared and some trends are noted.

\section{Low Luminosity AGN}

The most detailed studies of circumnuclear gas in AGN are available for nearby Seyfert galaxies. These observations reveal a continuous distribution of gas in disk-like structures from sub-parsec to kiloparsec scales via observations of ionised, atomic and molecular gas. In the centres of many objects the state of the gas is probably controlled by the ratio of hard X-ray heating from the central AGN to the local gas density (see Maloney, Hollenbach, \& Tielens 1996), giving an onion-skin structure with decreasing gas temperature with distance. The different gas components in rough order of distance are described in Sections 2.1 to 2.4. In sources with ongoing star-formation supernovae form an additional 
energy source which can modify the gas physical state on 100pc scales (see Section 2.5).

\subsection{Ionised Gas}

In NGC1068 $r<1$ pc VLBI continuum emission has been observed (Gallimore, Baum, \& O'Dea 1997); which it has been argued arises from thermal emission from the ionised inner edge of the nuclear torus. Searches for such emission in other Seyferts have been attempted with another candidate found (see Mundell et al.2000 and these proceedings). The ionised gas component can also be traced by means of free-free absorption. Pedlar et al. (1998) report free-free absorption on scales $r<50 \mathrm{pc}$ in NGC4151, while other cases of free-free absorption in Seyferts down to scales $r=1 \mathrm{pc}$ have been proposed by Wilson et al.(1998), Roy et al.(1998) and Ulvestad et al. (1999). It is unclear how much of the inferred free-free absorption occurs in highly ionised gas or is due to a small ionised fraction within an atomic gas component.

\subsection{Water Masers}

On scales $r=1-10 \mathrm{pc}$ in Seyferts circumnuclear water masers are sometimes observed. To date (Greenhill 2000 and these proceedings), circumnuclear water masers have been detected in about two dozen sources, all of them are Seyfert 2 galaxies or LINERS. The fact that there are no water masers in Seyfert 1 galaxies is consistent with Seyfert unification schemes. Only in four of the water masers so far studied (namely NGC4258, NGC1068, NGC4945 and Circinus) are the water maser spectra bright and complex enough that a full or partial circumnuclear disk is revealed by VLBI observations. The best example of this phenomena is still NGC4258. In NGC1068 and Circinus (Greenhill these proceedings) the maser disks appear to be substantially warped.

\subsection{Molecular Emission}

On scales $r=10-100 \mathrm{pc}$ the circumnuclear gas can be studied by millimetre molecular emission lines. Interferometric observations (Schinnerer et al. 2000) of CO in NGC1068 have probed gas on scales between $r=13 \mathrm{pc}$ and $r=100 \mathrm{pc}$ The gas kinematics on these scales have been modelled in terms of a warped disk. Single dish surveys suggest that dense gas tracers such as HCN are more abundant in Seyfert galaxies than normal spirals (Curran et al. 2000). Rapidly rotating $\mathrm{HCN}$ and $\mathrm{CO}$ gas has been imaged down to $\mathrm{r}=13 \mathrm{pc}$ in the Seyfert 1 galaxy NGC3227 (Schinnerer, Eckart, \& Tacconi 2000). In NGC3227 infrared observations (Fernandez et al. 1999) show emission from excited molecular hydrogen out to $r \approx 50 \mathrm{pc}$. The same authors also detect excited molecular hydrogen in NGC4151 at $r \approx 50 \mathrm{pc} 1998)$ just outside the inferred HI disk. In a few Seyfert galaxies the molecular gas dynamics on $r<100 \mathrm{pc}$ scales can be traced via observations of $\mathrm{OH}$ megamasers (see Section 2.5) but these only seem to occur in objects showing on-going starbursts.

\subsection{Atomic Absorption}

Gallimore et al. (1999) has summarised the observations of HI absorption in Seyferts. In most cases where the sites of HI absorption are mapped against 
the background continuum structures projected distances are $100 \mathrm{pc}$ to $600 \mathrm{pc}$ from the AGN core. In most cases the HI absorption avoids the central radio components. The HI column densities in the inner kiloparsec of Seyferts appear to be at least a factor of 10 greater than in inactive spirals and Gallimore et al speculate that differences in fuel supply in the inner $\mathrm{kpc}$ are required to trigger a Seyfert. These authors find a correlation between the inferred HI column density and the inclination of the host galaxy. If it is assumed that $\mathrm{HI}$ is in the galaxy plane it is possible to fit most $\mathrm{HI}$ absorption profiles, assuming inner disk radii of $10 \mathrm{pc}$ to $100 \mathrm{pc}$ and outer radii from $300 \mathrm{pc}$ to $1 \mathrm{kpc}$. NGC4151 seems to be an exception and requires a compact $r \approx 10 \mathrm{pc}$ disk. Although most HI absorption data in Seyferts is well fitted by obscuring disks, there are exceptions, in particular the highly blueshifted absorption in the southern Seyfert IC5063 (Oosterloo et al. 2000) which is well modelled by a jet-cloud interaction.

Gallimore et al. (1999) find in Seyferts no correlation between the inferred $\mathrm{HI}$ disk column densities and those deduced from X-ray photoelectric absorption observations. It is argued that the atomic gas spin temperatures are likely to be $100 \mathrm{~K}$, in which case the atomic column densities are also 10-100 times less than those derived from photoelectric absorption. Gallimore et al. conclude that most of the observed HI gas in Seyferts is associated with the inner part of the galactic disk rather than the region associated with the majority of the photoelectric column density. The finding that most of the HI absorption in Seyferts occurs on 500pc - 1000pc scales, in some cases outside the molecular emission, seems inconsistent with the expectation of decreasing gas excitation with radius (Maloney et al.1996). However depending on the gas density profile with distance, AGN X-ray heated gas can have atomic gas outside the molecular zone (see Neufeld, Maloney, \& Rees 1994). Alternatively at these large radii other non-AGN heating sources, i.e. stellar sources, might suffice to keep the gas atomic.

Various explanations are given by Gallimore et al. (1999) as to why (with the probable exception of NGC4151) there is no sign of any atomic absorption associated with the bulk of the photoelectric absorbing gas - given that models (Maloney et al. 1996) predict significant atomic gas between the radii where the gas is purely ionised and purely molecular. The possible explanations offered include line broadening, free-free absorption of the background continuum and radiative excitation of the atomic gas by the continuum. All three explanations require that the majority of the column density, and hence probably the majority of the optical obscuration, occurs on scales $r<10 \mathrm{pc}$.

\subsection{Nuclear Starbursts}

Radio observations have recently revealed large 100pc scale regions of continuum emission in three ultra-luminous infra-red galaxies, namely Mrk 231 (Carilli, Wrobel, \& Ulvestad 1998 and Taylor et al. 1999), Mrk 273 (Carilli \& Taylor 2000) and IC694 (Polatidis, this volume). The first of the above objects is a Seyfert 1 while the extent of the AGN component in the other two objects is unclear. In each case it has been argued that the radio continuum in these objects comes from synchrotron emission from relativistic electrons accelerated by supernovae which diffuse into the circumnuclear disk. It is argued that star formation rates of 50 - 200 solar masses per year are required to power this 
emission. In both Mrk 273 (Carilli \& Taylor 2000) and Mrk 231 (Taylor et al. 2000) compact candidate radio supernova remnants have been identified in VLBI maps, supporting this model. All these candidates have similar luminosities to the population of supernovae identified in Arp 220 by Smith et al. (1998). In all three objects high opacity HI absorption has been detected against the 100pc scale continuum (see references above), and velocity gradients measured which give estimates of enclosed masses. Additionally in Mrk273 (Yates et al. 2000), Mrk 231 (Richards this volume) and IC694 (Polatidis, this volume) OH megamasers are detected and have similar velocity gradients to the HI; suggesting that the regions of $\mathrm{OH}$ maser emission and atomic absorption partially overlap. These observations show that in AGN sources with active starbursts the physical state of the circumnuclear gas on 100 pc scales is likely to be greatly modified.

\section{Powerful AGN}

HST imaging (e.g. De Koff et al. 2000) has shown that nuclear dust structures on scales $<2 \mathrm{kpc}$ are common in powerful FRI and FRII radio-galaxies. In the former class of object the dust often has a settled disk morphology which is perpendicular to the the radio jet (perhaps the best example being the $r=$ 250pc disk in NGC4261, Jaffe et al.1993). In FRII radio-galaxies on the other hand a wider variety of dust morphologies, including disks, are seen. In the radio and millimeter the phenomenology of circumnuclear gas in powerful AGN is not quite as rich as for Seyferts. For instance despite extensive searches there have been no detections of water (Henkel et al. 1998) or OH mega-maser emission. Furthermore until recently searches for associated broad molecular absorption have not been very successful (see Section 3.3). Despite this there has been success in tracing the small scale ionised and atomic components of the circumnuclear gas on scales of $r<100 \mathrm{pc}$ as described in Sections 3.1 and 3.2 .

\subsection{Ionised Gas}

A number of detections of free-free absorption have been reported in radio loud AGN. The best studied cases so far are in NGC1275 (Walker et al. 2000) and NGC4261 (Jones et al. 2000 and these proceedings). The last reference gives a list of detected sources, to which recent detections in Compact Symmetric Objects (CSOs; doubles with overall size $<1 \mathrm{kpc}$ ) by Kameno et al. (2000) and Marr, Taylor, \& Crawford (2001) can be added.

In cases where free-free absorption is detected toward the cores of FRI or FRII sources, the object always shows a parsec scale counterjet as well as a jet. This is a remarkable result given the small number of two-sided parsec scale jets known. Invariably the free-free absorption is also concentrated against the counterjet feature. Both results are consistent with the free-free absorption occurring in an ionised disk orientated perpendicular to the radio jet. The results for the CSOs are also consistent with this model with usually only one minilobe being significantly free-free absorbed. Most ionised disk radii are estimated to be $\approx 1 \mathrm{pc}$, the smallest radius $(0.1 \mathrm{pc})$ occurring in the FRI NGC4261, and the largest radii $\approx 20 \mathrm{pc}$ occurring in CSOs. 
Another way to probe ionised gas (and magnetic fields) on small scales is via VLBI mapping of Faraday rotation of polarisation. This technique applied to the strongly polarised cores of core-dominated sources (Taylor 2000) shows evidence for an ionised gas component on scales of $r=1-100 \mathrm{pc}$.

\subsection{Atomic Absorption}

Flux limited surveys for atomic absorption in powerful AGN have been carried out by van Gorkom et al.(1989) and more recently by Morganti et al.(2001). In the sample of 29 sources surveyed by van Gorkom et al, 4 sources were detected in HI absorption, 3 of these could be classified as being dominated by Compact Symmetric (CSS) structure with overall size $<15 \mathrm{kpc}$. Morganti et al. (2001) detected 5 sources out of 23 surveyed; one of these sources had a CSS structure, another had a CSS structure embedded within a larger double. Of the remaining three sources one $\mathrm{HI}$ detection was toward the nucleus of a medium sized FRII source, one toward the nucleus of a FRI source and one toward a core-jet. Morganti et al. (2001) noted that the two galaxies in their sample with signs of on-going rapid star formation were both detected in HI absorption.

Despite the small number statistics compact CSS-like sources seem to be over-represented amongst the HI detections. This class of source only comprises $\approx 20 \%$ or so of sources selected in centimetre wavelength flux-limited surveys yet comprises $60 \%$ of the detections. It has been argued (Conway 1997) that the reason for this high detection rate is that these small radio sources all have their radio emission embedded within the dense nucleus of the host galaxy; and so provide good targets for detecting absorption. Consistent with this HI absorption observations targeted toward CSS sources show a high detection rate with possible evidence for a inverse correlation between source size and HI column density (Pihlström et al.2001).

VLBI HI absorption observations have been conducted toward HI absorbers in both CSS sources and the cores of twin jet FRII/FRI/LINER sources. Observations toward the former class include the small CSS sources 4C31.04 (Conway 1997) and 1946+708 (Peck, Taylor, \& Conway 1999) which both show absorption toward their minilobes and core components. In both cases the pattern of absorption can be fitted by an almost edge-on disk of radius $\approx 100 \mathrm{pc}$. HST observations of 4C31.04 and other CSOs have confirmed the presence of dust disks of the expected dimensions (Perlman et al. 2001). In contrast observations of the $2 \mathrm{kpc}$ scale CSS-like structure in $3 \mathrm{C} 236$ show that the absorption is associated with a jet-cloud interaction (Conway 2001). This observations shows that, as noted by Morganti et al (2001), obscuration by disks is not the only source of $\mathrm{HI}$ absorption.

Amongst the twin jet sources the results of VLBI HI observations have been presented for one FRII source (Cygnus A, Conway 1999), three FRI cores (Hydra A, Taylor 1996; 2322-123 Taylor et al. 1999b; NGC4261, van Langevelde et al. 2000), and one LINER NGC3994 (Peck et al. 1998). Remarkably all five objects show a detectable parsec-scale counterjet. Two other parsec-scale two-sided sources, 3C84 and NGC1052 also have broad HI absorption but have not yet been mapped by VLBI. Given the rarity of sources with parsec scale counterjets the correspondence with HI absorption is remarkable. This result can be understood if the HI absorption occurs primarily in a relatively thin 
disk structure which lies in a plane perpendicular to the radio jets. The atomic absorption is inferred to occur at between $r \approx 6 \mathrm{pc}$ (in NGC4261, van Langevelde et al. 2000) and $r \approx 50$ pc (in Cygnus A, Conway 1999).

\subsection{Molecular Absorption and Emission}

Both free-free and $\mathrm{HI}$ absorption observations provide evidence for circumnuclear gas in the plane perpendicular to the radio jets in FRII radio galaxies and are therefore consistent with orientation based unified schemes. In such schemes it is usually assumed that most of the obscuring column is molecular. However searches for broad CO and other molecular absorption toward the cores of FRIIs such as Cygnus A, which should be easily detectable, have up to now been largely negative (Drinkwater, Combes \& Wiklind, 1996). Evans et al. (1999) did recently detect $60 \mathrm{~km} / \mathrm{s}$ wide $\mathrm{CO}(1-0)$ absorption in the FRII radio galaxy 3C293 but this may well be an unusual object. There is evidence from the detected $\mathrm{CO}$ emission for a large $(3 \mathrm{kpc})$ massive circumnuclear disk in this object containing $10^{10}$ solar masses of gas. In this object the $\mathrm{CO}$ absorption may therefore be occurring some distance $(\approx 1 \mathrm{kpc})$ from the central engine. Note that in general searches for molecular emission in FRI or FRII sources have proved largely negative (see Evans et al. 2000), presumably because of the large distances to these sources and their usually relatively low molecular disk masses (see the estimates of, De Koff et al.2000). One exception however is the detection of a $r \approx 100 \mathrm{pc}$ CO disk in the nearby FRI Centaurus A (Rydbeck et al. 1993).

In contrast to emission the detectability of molecular absorption should be independant of distance and should be detectable. Several explanations have been given for the lack of such absorption toward Cygnus A and other classical FRII cores. Based on the detection of HI absorption it has been been proposed that the whole X-ray obscuring column is atomic (Conway \& Blanco 1995). Alternatively it has been suggested that radiative excitation of molecules due to nuclear millimetre continuum elevates the excitation temperature and makes absorption invisible (Maloney, Begelman \& Rees, 1994). Very recently Fuente et al. (2001) have in Cygnus A detected $\mathrm{CO}^{+}$absorption with similar velocity width to the HI absorption. It is argued that this molecular species can have relatively high abundance in X-ray irradiated circumnuclear gas - so that it is detectable despite radiative excitation effects. These observations show that molecular gas does indeed exist in the obscuring region of Cygnus A. Further observations of different transitions of the same ion should be able to localise at what radius the absorption occurs.

\section{Conclusions}

This review of the observations shows that in both low and high luminosity sources there is evidence for continuous distributions of gas from kiloparsec to parsec scales. The best examples in the two luminosity classes are respectively NGC1068 and NGC4261 (van Langevelde et al. 2000). HI absorption appears to occur at smaller radii in powerful sources than in Seyferts, this might be due to the former having more luminous hard X-ray sources compared to Seyferts. Alternatively the difference might be due to them having lower circumnuclear gas densities and so less cooling. Disks in FRI sources appear to be relatively 
thin (van Langevelde 2000, Morganti et al. 2001) which is consistent with recent optical results. The future combination of X-ray observations of the AGN heating sources, ALMA observations of molecular circumnuclear gas, HST/NGST observations of dust disks and central dark masses and radio interferometry will be a powerful one. This combination should lead us to a quantitative understanding of gas physical conditions versus radius in many AGN. This knowledge will in turn help us to constrain the mechanisms by which gas is fed into AGNs.

\section{References}

Carilli, C. L. \& Taylor, G. B. 2000, ApJ, 532, L95

Carilli, C. L., Wrobel, J. M., \& Ulvestad, J. S. 1998, AJ, 115, 928

Conway, J. E. 1997, 2nd Workshop on GPS and CSS Radio Sources, Leiden, Publ Leiden, Eds Snellan,I., Schilizzi,R.T., Rottgering,, H., \& Bremner M.

Conway, J. E., 1999, ASP Conf Series 156. 'Highly Redshifted radio Lines', 259 Conway, J. E., 2001, Proc 5th EVN Symposium, Publ Onsala Space Obs, Eds Conway, J.E., Polatidis, A., Booth, R.S., \& Pihlström, Y.M.

Conway, J. E. \& Blanco, P. E. 1995, ApJ, 449, L131

Curran, S. J., Aalto, S., \& Booth, R. S. 2000, A\&AS, 141, 193

De Koff, S., Best, P., Baum, S., Sparks, W., Rttgering, H., Miley, G, Golombek, D., Macchetto, F., Martel, A. 2000, ApJS, 129, 33

Drinkwater, M. J., Combes, F., \& Wiklind, T. 1996, A\&A, 312, 771

Evans, A. S., Sanders, D. B., Surace, J. A., Mazzarella, J. M. 1999, ApJ, 511, 730

Fernandez, B. R., Holloway, A. J., Meaburn, J., Pedlar, A., \& Mundell, C. G. 1999, MNRAS, 305, 319

Fuente, A., Black, J. H., Martin-Pintado, J., Rodriguez-Franco, A., GarcoaBurillo, S., Planesas, P., \& Lindholm, J., 2001, ApJ, in press.

Gallimore, J. F., Baum, S. A., O’Dea, C.P., Pedlar, A., \& Brinks, E. 1999, ApJ, 524,684

Gallimore, J. F., Baum, S. A., \& O'Dea, C.P. 1997, Nature, 388, 852

Greenhill, L. J. 2000, BAAS, 196, 2107

van Gorkom, J. H., Knapp, G. R., Ekers, R. D., Ekers, D. D., Laing, R. A., \& Polk, K. S. 1989, AJ, 97, 708

Henkel, C., Wang, Y. P.,Falcke, H., Wilson, A. S., \& Braatz, J. A. 1998, A\&A, 335,463

Jaffe, W., Ford, H.C., Ferrarese, L., van den Bosch, F., O'Connell, R. W., 1993, Nature, 364, 213

Jones, D. L., Wehrle, A.E., Meier, D. L., \& Piner, B. G., 2000, ApJ, 534, 165

Kameno, S., Sawada-Satoh, S., Shibata, K. M., Inoue, M., Wajima, K. 2000, Astrophysical Phenomena Revealed by Space VLBI, Proc of the VSOP Symposium, Eds.: H. Hirabayashi, P.G.Edwards, and D.W. Murphy, Publ ISAS, Japan. 87 
van Langevelde, H. J., Pihlström, Y. M., Conway, J. E., Jaffe, W., \& Schilizzi, R. T. 2000, A\&A, 354, L45

Maloney, P. R., Begelman, M. C., \& Rees, M. J. 1994, ApJ, 432, 606

Maloney, P. R., Hollenbach, D. J., \& Tielens, A. G. G. M. 1996, ApJ, 466, 561

Marr, J. M., Taylor, G. B., \& Crawford, F., III, 2001, ApJ, accepted.

Morganti, R., Oosterloo, T. A., Tadhunter, C. N., van Moorsel, G., Killeen, N., \& Wills, K. A., 2001 MNRAS, in press.

Mundell, C. G., Wilson, A. S., Ulvestad, J. S., \& Roy, A. L. 2000, ApJ, 529, 816 Neufeld, D. A., Maloney, P.R., \& Rees, M.J. 1994, ApJ, 447, L17

Oosterloo, T. A., Morganti, R., Tzioumis, A., Reynolds, J., King, E., McCulloch, P., \& Tsvetanov, Z., 2000, AJ, 119, 2085

Peck, A. B., \& Taylor, G. B. 1998, ApJ, 502, L23

Peck, A. B., Taylor, G. B., \& Conway, J. E. 1999, ApJ, 521, 103

Pedlar, A., Fernandez, B., Hamilton, N. G., Redman, M. P., \& Dewdney, P. E., 1998, MNRAS, 300,1071

Perlman, E. S, Stocke, J. T., Conway.J.E., \& Reynolds,C. 2001, AJ, submitted

Pihlström, Y. M., Vermuelen, R. C., Conway, J. E., \& Taylor, G. B. 2001., A\&A, in preparation

Roy, A. L., Colbert, E. J. M., Wilson, A. S., \& Ulvestad, J. S. 1998, ApJ, 504, 147

Rydbeck, G., Wiklind, T., Cameron, M., Wild, W., Eckart, A., Genzel, R. \& Rothermel., H., 1993, A\&A, 270, L13

Schinnerer, E., Eckart, A. Tacconi, L. J., Genzel, R., \& Downes, D. 2000, ApJ, 533,850

Schinnerer, E., Eckart, A., \& Tacconi, L. J. 2000, ApJ, 533, 826

Smith. H. E., Lonsdale, C. J. Lonsdale, C. J., \& Diamond, P. J. 1998, ApJ, 493, L17

Taylor, G. B. 1996, ApJ, 470, 394

Taylor, G. B.; Silver, C. S., Ulvestad, J. S., Carilli, C. L. 1999, ApJ, .519, 185

Taylor, G. B. 2000, ApJ, 533, 95

Taylor, G. B., Silver, C. S., Ulvestad, J. S., \& Carilli, C. L. 1999a, ApJ, 519, 185

Taylor, G. B., O'Dea, C. P., Peck, A. B., \& Koekemoer, A. M. 1999b, ApJ, 512, L27

Ulvestad, J. S., Wrobel, J. M., Roy, A. L., Wilson, A. S., Falcke, H., \& Krichbaum, T. 1999, ApJ, 517, L81

Walker, R. C., Dhawan, V., Romney, J. D., Kellermann, K. I., \& Vermeulen, R. C. 2000, ApJ, 530, 233

Wilson, A. S., Roy, A. L., Ulvestad, J. S., Colbert, E. J. M., Weaver, K. A., Braatz, J. A., Henkel, C., Matsuoka, M., Xue, S., Iyomoto, N., \& Okada, K. 1998, ApJ, 505, 587

Yates, J. A., Richards, A. M. S., Wright, M. M., Collett, J. L., Gray, M. D., Field, D., \& Cohen, R. J. 2000, MNRAS, 317, 28 